\title{
Implementing the care programme approach in psychotherapeutic settings
}

\author{
Chris Mace
}

\begin{abstract}
The care programme approach (CPA) should be implemented across all mental health services. However, the specific needs of psychotherapeutic settings were not recognised in the official documentation. The scope for conflict between therapeutic practices and CPA requirements may have increased following revision of CPA guidelines. A recent survey of consultants in psychotherapy confirmed that implementation of CPA remained uneven. The additional time required, threats to confidentiality and a negative impact on the therapeutic relationship were common concerns. Positive effects on assessment procedures and liaison between services were also reported. Practical suggestions for minimising potential negative effects of CPA are given. It is recommended that all services providing psychological therapies develop a written policy for the local implementation of CPA.
\end{abstract}

The care programme approach (CPA) has been recommended practice in mental health services since 1990 (Department of Health, 1990). Its key principles (Box 1) were summarised in plain language in a report by the Department of Health (2001a).

The implementation of care programming presents a number of complex challenges. Patients whose special needs are obvious, by virtue of their dependence on others by reason of age or incapacity, have already received attention (e.g. Roy, 2000; Philpot et al, 2001). However, the thousands of people whose contact with mental health services results in their receiving talking treatments from professionals trained to provide these have received virtually no attention in the literature to date. The assumption is that care programming is straightforward in psychotherapeutic settings. This article examines where tensions do in fact occur between the agendas of CPA and psychotherapy, and how these might be reduced in practice.

\section{The range of psychotherapeutic settings}

All mental health services are, potentially, psychotherapeutic settings, in that there is always scope for the relationships between patients and

\section{Box 1 Principles of the care programme approach (after Department of Health, 2001a)}

Everyone accepted for treatment or care by mental health services should have:

- their needs for treatment and care assessed

- a package of care (care plan) to meet those needs drawn up

- a named mental health worker (keyworker or care coordinator) to keep in close touch with them

- a regular review of their needs and their care plan

professionals (and between patients meeting together) to assist or impede recovery. This article focuses on settings where psychotherapy is the primary treatment offered: these include out-patient services where planned talking treatments are provided individually or to groups or families, as well as milieux such as psychotherapy day hospitals or therapeutic communities in which several staff members are likely to remain directly involved in each patient's treatment. In all of these settings, a confiding working relationship is provided, in which high levels of disclosure and skilled support are expected, irrespective of the therapeutic model that is followed.

Chris Mace is consultant psychotherapist to the South Warwickshire Primary Care Trust (Ashton House, 15 George Street, Leamington Spa, Warwickshire CV31 1ET, UK), where he has been Clinical Manager of the Psychodynamic Psychotherapy Service since 1992. He is also an honorary senior lecturer in psychotherapy at the University of Warwick. His current research interests include clinical assessment for psychotherapy, risk assessment and the analysis of obstacles to care within publicly funded services. 
Box 2 Key revisions of the care programme approach (after Department of Health, 1999a)

- CPA is integral to care management

- There is a unified process for planning, review and risk management

- The approach applies regardless of setting

- One person coordinates all care

- There is a single access route to services

- There are common information and audit requirements

- 'Standard' and 'enhanced' packages are differentiated

- Support is provided for carers as well as for service users

\section{Care programming}

Since the original principles summarised in Box 1 were established, guidance on the implementation of care programming has been updated in two publications by the Department of Health $(1999 a, b)$ : Effective Care Coordination in Mental Health Services (Box 2) and the National Service Framework for Mental Health (Box 3).

Effective Care Coordination in Mental Health Services reintroduced care programming as a universal system of case management to coincide with the harmonisation of procedures between health and social services care, and the abolition of case registers for patients at greatest risk. It increased the responsibility of named keyworkers to encompass all aspects of care. Two levels of care programming, 'standard' and 'enhanced', were differentiated for the first time. The latter was likely to apply if multiagency input was required in order to reduce risk in relevant areas. Detailed guidance was provided concerning the criteria that should determine which level of care was given; care coordinators' responsibilities in either situation; and the collection and sharing of information. The document included illustrative vignettes as well as bald statements of the measures that should be taken. Subsequent guidance has confirmed timetables for full implementation of CPA and a national audit framework to monitor this (Department of Health, 2001a).

Despite this clarification, there has been scope for confusion. Effective Care Coordination in Mental Health Services makes plain the difference between standard and enhanced packages, and states:

'For those requiring standard CPA (this might include those who need the support of only one agency or discipline) it is only necessary for professionals to maintain adequate clinical/practice records which record the assessment of the service user's needs, the agreed care plan and the date of the next review of the care plan. Elements of risk and how the care plan manages the identified risk must always be recorded' (Department of Health, 1999a: para. 63).

Contradictions between this and Standard 4 of the National Service Framework for Mental Health (Box 3) can be reconciled by assuming that the latter is referring to 'enhanced' care programming only. However, in a later summary (Department of Health, 2001c), it is explicitly stated that requirements that patients on enhanced CPA should have written plans to secure suitable employment, adequate housing and appropriate welfare benefits 'will apply to everyone on CPA' by March 2004.

Whether the guidance on CPA has been consistent or not, what specific comments does it make about psychotherapy? Effective Care Coordination in Mental Health Services makes little reference to the 'care' that patients actually receive. This is something that professionals provide, but there is not a single reference to a mental health professional as someone who might provide 'therapy' rather than 'support'. An appendix provides eight vignettes to illustrate implementation of the CPA in practice. These are clear and instantly recognisable, but again contain no reference to a patient receiving psychotherapeutic help within mental health services. Two patients who need psychological interventions are denied mental health services as they do not 'need to be subject to the $\mathrm{CPA}^{\prime}$ - one of these is a middle-aged man who is actively seeking help. He is losing his job, has stomach pains from excessive drinking and is now afraid he is dying following the onset of panic attacks. It is recommended he should be offered selfhelp literature and his general practitioner should be offered 'support'. The advice on two other patients, candidates for psychological interventions

Box 3 National Service Framework for Mental Health: Standard 4 (after Department of Health, 1999b)

All mental health service users on CPA should:

- receive care that optimises engagement, anticipates or prevents crises, and reduces risk

- have a copy of a written care plan that describes the action to be taken in a crisis by the service user, the carer, and the care coordinator; advises the service user's general practitioner how to respond if the user needs additional help; and is regularly reviewed by the care coordinator

- be able to access services 24 hours a day, 365 days a year. 
according to current treatment guidelines (Department of Health, 2001c; National Institute for Clinical Excellence, 2002), is as follows. A woman with a history of repeated self-cutting who discloses sexual abuse 'may benefit from intensive support under enhanced CPA' - but not psychotherapy (Department of Health, 1999a: case 1). A man whose disturbed behaviour is associated with hearing voices has a complex care package spelled out in which the principal role of mental health services is 'for monitoring of his mental state and for supervision of his medication' (case 5). That the place of psychotherapeutic interventions within mental health services is ignored or denied here suggests that their impact on the implementation of care programming received little attention when the policy was 'modernised'. Perhaps the contribution of psychological therapists to care would have been reflected more accurately in policy on care programming if some of the principles of CPA - meeting to agree a plan and to review it in the light of developments had applied during its own planning.

\section{Psychotherapy and care planning}

Why should the requirements of care programming present any 'challenges' to psychotherapeutic practice? Even if the role of psychological therapies in mental health services has been insufficiently recognised, it is not evident that conflicts need arise between the demands of psychotherapeutic work and care programming, given the statement of principles in Box 1. As Box 4 illustrates, it is possible to state a parallel set of principles of good practice that would apply across the psychotherapies, irrespective of the psychotherapist's favoured model (psychodynamic, cognitive-behavioural or systemic, for example).

When framed in this way, there is a clear correspondence between Box 1 and Box 4 . These principles of therapeutic practice were being widely

Box 4 Four principles of good therapeutic practice

- Needs and vulnerabilities are assessed before treatment is begun

- Treatment is founded on a careful formulation

- A single therapist sees treatment through to its conclusion

- Clinical progress and the working formulation are regularly reviewed through supervision observed long before CPA was introduced or the phrase 'reflective practice' was devised (the latter effectively describes the transfer of basic therapeutic disciplines to other clinical arenas). If care programming in psychotherapeutic settings was simply about applying the principles in Box 1, conflict should be less than in arenas where reflective practice had been less familiar.

However, it is clear from Box 2 that 'effective' care programming has been expected to achieve more than organisation of individual treatments. In essence, care programming has moved from being a tool in the delivery of clinical care to become a prescription for how a range of systems should be coordinated. With this revision, three additional major objectives are apparent, namely integration of each individual's health and social care; streamlining of information systems; and responding to carers' needs. Each of these is potentially problematic in psychotherapeutic settings, especially if the current expectations for 'enhanced' CPA were extended to all patients.

\section{Integration of health and social care}

The first objective involves assumption of an administrative role (as care coordinator between services), which is not traditional for psychotherapists. This could make psychotherapists responsible for convening review meetings that involve other professionals and carers as well as their patient. Even if a patient's psychotherapist is not that patient's care coordinator, the pursuit of integration will create expectations that the psychotherapist will attend and contribute to meetings convened by other mental health professionals or social workers. Both situations can challenge the psychotherapist's attempts to establish and maintain a working relationship that is privileged in its exclusion of others and in the confidences that may be shared. There is potential conflict here between procedures appropriate to psychotherapy assessment and those required for CPA. For example, questions about employment, housing and financial circumstances would only normally be pursued if they were relevant to decisions about future therapy. To introduce them in a first interview could mislead a patient about what the assessor considers to be important.

\section{Streamlining information systems}

The second objective, that of streamlining information systems, is also relevant to clinical assessment. Protocols to assess areas such as risk according to a standardised format may make no 
concessions to the setting, in terms of the kind of risks that are routinely enquired into or the questions that are expected to be put. This is at odds with some assessment practice, especially in the psychodynamic therapies. The assessor might wish the interview's structure to remain fluid in a deliberate and carefully modulated way, so that inferences can be drawn about therapeutic needs from the way patients conduct themselves in an intentionally open situation. Equally, assessing therapists may wish to remain free to respond to whatever the patient might introduce to the interview. If, instead, patients are faced with an introductory barrage of set questions about, say, possible homicidal intent, suspicions of the assessor will be prompted that would severely restrict the interview's usefulness in this respect.

Apart from the possible implications for what therapists are expected to do and think about, inclusion of psychotherapists in attempts to unify information systems can conflict with codes of professional practice. Plans to format clinical information so that it might be available, on some 'need to know' basis, to professionals in remote care systems without the service user's active consent are likely to conflict with psychotherapists' ethical duty to safeguard the very personal disclosures that are often made to them, on their patients' behalf.

\section{Responding to carers' needs}

The third objective, paying attention to carers' needs, has already been touched on in the comments about review meetings. The spirit of Effective Care Coordination in Mental Health Services is that carers would often be involved from the outset in assessment of their relative's needs and planning treatment. As with the other objectives, the potential challenge here is greater for some forms of psychotherapy than others. Although behavioural therapists have enlisted family members as cotherapists with success, psychodynamically trained psychotherapists usually minimise contact with relatives and other carers in order to focus on the inner world of projections and fantasies their patients present. Although systemic therapists expect to work with family systems and even complete families, sharp divisions between 'user' and 'carer' are antithetical to attempts to help family members recognise shared responsibilities for joint problems.

These comments are far from exhaustive, but they indicate how there is a good deal of scope for friction between the implementation of CPA, particularly that designated as 'enhanced' CPA, and many psychotherapists' working practices. Is this potential for conflict realised in practice?

\section{A brief survey of CPA implementation}

To assess the current implementation and impact of CPA in psychotherapy, in January 2003 I conducted a survey among 20 psychotherapy consultants from different services. The majority worked wholly in dedicated out-patient services, but three consultants from therapeutic communities and two from general psychiatry also responded. Two consultants worked in Scotland, where legislative requirements are different. Only one service reported that cognitivebehavioural therapy rather than psychodynamic psychotherapy was its principal model.

Of these 20 services, 14 reported having implemented CPA for patients receiving psychotherapy. No two services appeared to be doing this in exactly the same way. Only half had a servicespecific written policy outlining how implementation should be done. The actual adoption of care planning appeared to be rather lower than that reported: only 12 services reported having a written care plan for each patient, and this figure included two services that had reported themselves to be noncompliant with CPA, but used written plans for internal reasons. If evident compliance is equated instead with staff being designated as care coordinators, at least for standard CPA, then 11 services were using CPA. This group included all 10 of the services that said they had implemented CPA and were writing care plans.

Additional questioning about clinical records and staff roles revealed further variations in practice. Among the 14 services that said they used CPA, 10 reported having a summary care plan for each new patient, 8 also used a structured risk assessment, 5 used a structured clinical history, and 4 made a routine record of housing and financial circumstances. Although 11 services said that staff members acted as care coordinators, only 6 said that staff members ever acted as coordinators for enhanced CPA. Only one service reported that trainees or other non-permanent staff acted as care coordinators.

The only service that claimed to involve relatives routinely in drawing up care plans was the one offering cognitive-behavioural therapy. When the procedure for review of care plans was enquired about, it was evident only 5 of the 14 services claiming CPA had a distinct review process involving the patient.

\section{Current attitudes to CPA}

All respondents were asked to comment on whether they felt care programming had improved the care received by patients. Six said that it had, two said 
Box 5 Advantages of the care programme approach

Perceived advantages of CPA were that it:

- ensured that clinical decisions were made and were explicit

- ensured that risks were recorded

- improved liaison between professionals

- clarified staff roles

- led to greater recognition by managers of the importance of the service

they were unsure, leaving a majority reporting no benefit or a net negative impact. When asked to list up to three advantages and/or disadvantages, most of the observations made were repeated by other members of the sample (Boxes 5 and 6 summarise the most common responses).

Perceived advantages were that CPA ensured that clinical decisions were made and that they were explicit. The outcome of an assessment would be stated and subsequent action set out. One consultant also commented on the value of being prompted to make statements about expected outcomes in the evaluation of a service. Others conceded that formalising risk assessment was helpful in ensuring it was completed. Another group of comments concerned the benefits of CPA for improving liaison between professionals. Care programming did ensure that psychotherapists were invited to multidisciplinary meetings. Although this was not universally welcome (because of perceived conflicts with the therapeutic stance), several consultants reported using these invitations as an opportunity to help colleagues think more dynamically about patients they shared. At a more mundane level, respondents welcomed the fact that at least a minimal level of communication and consultation was expected to take place, even if it was not necessarily guaranteed.

Among other points, clarification of roles through care programming was felt to be helpful not only operationally, but also in reducing some professionals' anxiety. One consultant observed that enrolment of psychotherapy patients on CPA had been politically beneficial, in helping the severity of their needs to be recognised and the service's contribution to be counted by managers. Another consultant, who was particularly concerned about the impact of CPA on patients joining a therapeutic community, commented favourably on its usefulness in engaging clients initially and in pre-emptive discharge planning.

The most commonly cited disadvantage was the sheer demand on time of additional bureaucracy and form-filling. Two consultants commented specifically on the extent to which this could reduce the availability of nurse therapists within clinical teams, and delays in discharge from a therapeutic community were attributed to CPA requirements. The second most frequent comment concerned patient confidentiality: CPA was perceived as leading to a centralisation of detailed clinical data that was antithetical to patients' interests. One respondent commented specifically on the potential impact of a record of having been 'CPA' $\mathrm{d}$ ' on the patient's future insurance and financial assessments.

Several respondents commented on conflicts between the particular demands of CPA assessment for prospective patients and the established processes of the psychotherapy service. Patients were given an inappropriate introduction to therapeutic thinking, if they were being asked all kinds of questions that would not be required for a clinical assessment. Some respondents believed that this could alienate new patients and dissuade them from proceeding. Consultants reported conflict for themselves between the demands of CPA assessment and maintenance of an 'analytic attitude'. Some expressed clear hostility - the procedures of CPA were not only reportedly redundant but 'antithought'.

As with potential benefits, some perceived disadvantages were mentioned only by the consultants working in therapeutic communities, reflecting apparent conflict with their therapeutic philosophy. The individualistic nature of care programming could clash with a philosophy in which all treatment occurred through groups. Moreover, CPA was believed to encourage community residents to think others were taking responsibility for things they should be taking responsibility for themselves. Even the language of 'risk assessment' was at odds with a policy of responsible 'risk minimisation'.

Overall, responses did reflect clear differences in working context. Both consultants who also worked substantially in general psychiatry were positive about the benefits of CPA, which they appeared to

Box 6 Disadvantages of the care programme approach

Perceived disadvantages of CPA included:

- time involved in form-filling

- risks to confidentiality from centralising data storage

- assessment questions send wrong message to patients

- conflict with philosophy of care 
value in the latter context. This underlines the extent of negative perceptions among the remaining fulltime psychotherapists. Curiously, views between consultants working with therapeutic communities were sharply divided. They all appeared to recognise the needs for structure, communication and open documentation, but disagreed about whether CPA had anything to offer in meeting these needs.

\section{Living with CPA}

Many participants in the survey took up an invitation to comment on measures they had found personally helpful in accommodating care programming. Most of these concerned ways to minimise its negative effects through avoidance, at either institutional or personal levels. Institutionally, three opt-out positions were reported. First, some consultants had simply made no attempt to alert local managers to their lack of interest in implementing CPA, and had not as yet been asked to introduce the framework. Other consultants had fought down attempts to introduce CPA, using arguments about its inappropriateness for their client population and treatment philosophy, and had won agreements that their service should be exempt. A third position held that psychotherapy services had so much in common with primary care services that they should be considered to belong with them for CPA purposes. (For brevity, I have called these the 'ostrich', 'lion' and 'chameleon' positions.)

Three further positions amounted to restricted implementation. First, the survey findings that only half the services implementing CPA were providing care coordination for patients on enhanced CPA indicate that the restriction of this responsibility to patients on standard CPA is a common policy. Second, systematic avoidance of the care coordinator role was sought through referral protocols that favoured prospective patients from within the secondary system (these being likely to have a coordinator already); however, there appeared to be some risk of services assuming a pseudo-tertiary status, which imposed barriers to access if this strategy was adopted. Third, there is the position of the (mainly residential) settings in which responsibility for care planning is returned to the patient. The patient draws up an appraisal and proposal for treatment goals which is then signed by staff, rather than the other way round. Its scope in out-patient settings appears to have been relatively untested.

In terms of individual strategies to minimise conflicts and additional burden, the likely consequence of becoming a care coordinator was reported to have made the task of assessment of new patients less attractive within some psychotherapeutic settings. Conversely, from reports from some residential and out-patient services it was evident that a senior figure such as the consultant psychotherapist had come forward to act as coordinator for many patients simultaneously, enabling other staff to maintain a purely therapeutic role.

Where the role of care coordinator was not avoided, a number of strategies for making it more acceptable were reported. These included simplification of paperwork - so that summary letters served as care plans, as the passage quoted above from Effective Care Coordination in Mental Health Services recommends for standard CPA. A useful suggestion for conducting standardised risk assessment concerned use of the risk sub-scale of the Clinical Outcomes in Routine Evaluation (CORE) questionnaire when this is routinely used with new patients - a practice whose validity in detection of risk is supported by evidence from a specialist psychotherapy setting (Whewell \& Bonanno, 2000).

Simplification of paperwork was also a way of reducing concerns over confidentiality aroused by the streamlining of information systems. It was interesting that so few services had electronic systems for pooling CPA data, but concerns on this score seem likely to increase with time. A more general point is apparent, that it is likely to be beneficial for psychotherapy teams to establish their own policy for implementation of CPA so that this can respect local needs in ways that a policy for the whole health care trust might not.

As the results of the survey indicate, few services have changed their practice to involve carers more meaningfully in planning and review. Indeed, because of the availability of parallel supervision or team meetings, reviews to formally satisfy CPA requirements were often literally a paper exercise, rather than an additional meeting with the patient.

\section{Conclusions}

The care programming approach has evolved into a system of case management that has not specifically taken the needs of psychotherapeutic settings into account. Although the basic philosophy of proper assessment and planned care represents little threat to psychotherapeutic work, secondary demands for broader assessment, information sharing and carer involvement can lead to genuine tensions. It is possible to realise some potential benefits and minimise disadvantages by establishing a clear service policy that takes the local circumstances and individual needs of a dedicated psychotherapy setting into account. 


\section{References}

Department of Health (1990) The Care Programme Approach for People with a Mental Illness Referred to the Specialist Psychiatric Services (HC(90)23). London: Department of Health.

Department of Health (1999a) Effective Care Coordination in Mental Health Services: Modernising the Care Programme Approach. London: Department of Health.

Department of Health (1999b) National Service Framework for Mental Health: Modern Standards and Service Models for Mental Health. London: Department of Health.

Department of Health (2001a) An Audit Pack for Monitoring the Care Programme Approach. London: Department of Health.

Department of Health (2001b) The Journey to Recovery - The Government's Vision for Mental Health Care. London: Department of Health.

Department of Health (2001c) Treatment Choice in Psychological Therapies and Counselling: Evidence Based Clinical Practice Guideline. London: Department of Health.

National Institute for Clinical Excellence (2002) Schizophrenia: Core Interventions in the Treatment and Management of Schizophrenia in Primary and Secondary Care. London: NICE.

Philpot, M., Hales, H., Sheehan, B., et al (2001) The use of operationalised criteria for inclusion on a Care Programme Approach register. Psychiatric Bulletin, 25 $172-174$.

Roy, A. (2000) The Care Programme Approach in learning disability psychiatry. Advances in Psychiatric Treatment 6, 380-387.

Whewell, P. \& Bonanno, D. (2000) The Care Programme Approach and risk assessment of borderline personality disorder: clinical validation of the CORE risk sub-scale. Psychiatric Bulletin, 24, 381-384.

\section{Multiple choice questions}

1 CPA requires:

a psychotherapy to be provided by a multidisciplinary team

b relatives to be told the outcome of a patient's assessment

c a risk assessment to be recorded

d regular reviews to be conducted

e employment counselling to be offered.
2 Conflicts can arise with therapeutic objectives because:

a care plans should be shared with patients

$\mathrm{b}$ assessment protocols may cover social needs

c care coordinators' responsibilities exceed those of therapists

d patients' dependency may be fostered

e CPA increases patients' symptoms.

3 Strengths of care programming in therapeutic settings include:

a enhancement of training

$\mathrm{b}$ clarification of roles

c prevention of users' complaints

d formal risk assessment

e communication with other services.

4 Many psychotherapists are wary of care programming because:

a patient confidentiality can be threatened

$b$ it exposes them to litigation

c they are invited to meetings

$d$ its principles are misguided

e it requires additional time.

5 Conflicts arising from CPA implementation might be reduced by:

a using electronic forms

b prescribing more selective serotonin reuptake inhibitors

c having a service-specific CPA policy

d postponing implementation

e employing a data clerk.

\section{MCQ answers}

\begin{tabular}{|c|c|c|c|}
\hline 1 & 2 & 3 & 4 \\
\hline $\mathrm{F}$ & a $F$ & a $\mathrm{T}$ & a $\mathrm{T}$ \\
\hline $\mathrm{F}$ & b $\mathrm{T}$ & b $\mathrm{T}$ & b F \\
\hline $\mathrm{T}$ & c $\mathrm{T}$ & c F & c F \\
\hline $\mathrm{T}$ & $\mathrm{d} T$ & $\mathrm{~d} T$ & $\mathrm{~d} \mathrm{~F}$ \\
\hline $\mathrm{F}$ & e $F$ & e $\mathrm{T}$ & e $\mathrm{T}$ \\
\hline
\end{tabular}

